\title{
Amygdalar Interaction with the Mediodorsal Nucleus of the Thalamus and the Ventromedial Prefrontal Cortex in Stimulus-Reward Associative Learning in the Monkey
}

\author{
David Gaffan 1 and Elisabeth A. Murray ${ }^{2}$ \\ 'Department of Experimental Psychology, Oxford University, Oxford OX1 3UD, England, and 'Laboratory of \\ Neuropsychology, National Institute of Mental Health, Bethesda, Maryland 20892
}

\begin{abstract}
Cynomolgus monkeys (Macaca fascicularis) were assessed for their ability to associate visual stimuli with food reward. They learned a series of new 2-choice visual discriminations between colored patterns displayed on a monitor screen. The feedback for correct choice was the delivery of food. In order to promote associative learning between the visual stimuli and the incentive value of the food reward, reward delivery was not accompanied by any distinctive visual feedback on the display screen. The rate of learning new problems was assessed before and after surgery in a total of 16 monkeys. Three groups of 3 monkeys received bilaterally symmetrical ablations in either the amygdala, the mediodorsal nucleus of the thalamus, or the ventromedial prefrontal cortex. All these groups showed a severe postoperative learning impairment. Seven other animals were given a unilateral ablation in 1 of those 3 structures and a second unilateral ablation, in each case contralateral to and different from the first, in order to produce 2 groups in which a putative amygdalo-thalamo-prefrontal pathway had been disconnected by crossed unilateral lesions. One disconnection group had lesions in the amygdala and ventromedial prefrontal cortex; the other had lesions in the amygdala and the mediodorsal nucleus of the thalamus. The disconnection groups showed a significant impairment, but the effect of the disconnection surgeries was significantly milder than the effect of any of the 3 bilaterally symmetrical lesions. Therefore, symmetrical bilateral lesions in either the amygdala, the mediodorsal nucleus, or the ventromedial prefrontal cortex produce similar impairments in the present task, implying that these structures are functionally related to each other; but the relatively mild effect of disconnecting these structures from each other argues against the hypothesis that they are serial stages in a single, tightly linked functional pathway.
\end{abstract}

\footnotetext{
Received Jan. 17, 1990; revised June 6, 1990; accepted June 8, 1990.

The research was supported by the Medical Research Council and by NATO Grant $0184 / 87$. We thank S. Watkins for assistance in training the monkeys. Send reprint request to either D. Gaffan, Department of Experimental Psychology, South Parks Road, Oxford OX1 3UD, England, or E. A. Murray, Laboratory of Neuropsychology, National Institute of Mental Health, Building 9, Room 1N-107, Bethesda, MD 20892.

Correspondence should be addressed to D. Gaffan, Department of Experimental Psychology, South Parks Road, Oxford OX1 3UD, England.

Copyright (C) 1990 Society for Neuroscience $0270-6474 / 90 / 113479-15 \$ 03.00 / 0$
}

One kind of associative learning in which the amygdala is of central importance is the association of a sensory stimulus with the incentive value of food reward. In visual-discrimination learning for food reward, the role of the amygdala varies according to the associative structure of the task. If visual discriminative stimuli are associated directly with the incentive value of the food reward, efficient learning depends on an intrahemispheric interaction between the amygdala and the visual-association cortex ipsilateral to it (E. A. Gaffan et al., 1988). But when visual discriminative stimuli are associated with the incentive value indirectly, via the mediation of a direct association with an auditory or visual secondary reinforcer, visual interaction with the amygdala is less important (Gaffan and Harrison, 1987; Gaffan et al., 1989).

In the present experiment, we examined a task in which visual discriminative stimuli are associated directly with the incentive value of the food reward. Our aim was to determine which structures, apart from the visual-association cortex, the amygdala interacts with in this task. E. A. Gaffan et al. (1988) showed that this type of learning is severely impaired by crossed unilateral lesions of the amygdala in one hemisphere and of the visual-association cortex in the other hemisphere. This combination of lesions disconnects the intrahemispheric pathway from the visual-association cortex to the amygdala by destroying its origin in one hemisphere and its destination in the other. The results suggested that visual information and incentivevalue information converge in the amygdala and are associated together there. If so, then the product of this associative process (the information, retrieved from memory, that a certain visual stimulus has been paired in the past with food) needs to be rclayed to further projection targets in order to elicit ultimately a motor response, namely, an instrumental discriminative response to that stimulus. Possible stages of this further pathway can be tested by disconnection experiments. Disconnection of the amygdala from further projection targets in the hypothetical pathway should disrupt the present type of visual associativelearning task in the same way as does disconnection of the amygdala from visual-association cortex.

The amygdala and adjacent periallocortical regions send a direct ipsilateral projection to the medial part of the mediodorsal thalamus (Porrino et al., 1981; Aggleton and Mishkin, 1984; Russchen et al., 1987; Gower, 1989). The medial part of the mediodorsal thalamus, in turn, projects heavily to the ipsilateral ventromedial prefrontal cortex (Tobias, 1975; Goldman-Rakic and Porrino, 1985; Giguere and Goldman-Rakic, 1988). Bache- 
valier and Mishkin (1986) have argued from behavioral results in recognition memory tasks that "the medial temporal, medial thalamic and ventromedial prefrontal regions of the brain are each apparently part of an integrated memory circuit" (p 260). In the present task, therefore, a simple and attractive hypothesis is that information and control flows from the visual discriminative stimulus, associated in memory with food reward, to the instrumental discriminative response along a tightly linked pathway through the visual-association cortex, amygdala, thalamus, and prefrontal cortex. If this hypothesis is true, then bilaterally symmetrical lesions in any of these structures should disrupt this type of learning. Furthermore, crossed unilateral lesions of the amygdala and ventromedial prefrontal cortex, or of the amygdala and mediodorsal thalamus, should disrupt learning as effectively as bilateral lesions in each of these structures, because they should disconnect the pathway of the flow in the same way as the crossed unilateral lesions of the amygdala and visual association cortex did in the experiment by E. A. Gaffan et al. (1988).

Therefore, we compared the learning disability produced by bilateral lesions with the learning disability produced by disconnection of the hypothesized pathway. One disconnection group had crossed unilateral lesions of the ventromedial prefrontal cortex and the amygdala; in addition, this group was subsequently tested, after forebrain commissurotomy, for the possibility that their residual learning ability was mediated by interhemispheric pathways. Another disconnection group had crossed unilateral lesions of the amygdala and mediodorsal thalamus. These 2 disconnection groups were compared with 3 groups having bilaterally symmetrical lesions in the amygdala, mediodorsal thalamus, or ventromedial prefrontal cortex. The lesions of the ventromedial prefrontal cortex were designed to remove the part of the prefrontal cortex that receives the heaviest projection from the medial part of the mediodorsal nucleus of the thalamus.

In addition to the projection relayed via the mediodorsal thalamus, the ventromedial prefrontal cortex receives a direct projection from the amygdala (Porrino et al., 1981; Amaral and Price, 1984). If this direct projection is of functional importance in the present task, crossed unilateral lesions of amygdala and ventromedial prefrontal cortex should have a greater effect than crossed unilateral lesions of the amygdala and mediodorsal thalamus. Similarly, bilateral ventromedial prefrontal lesions should have a greater effect than bilateral mediodorsal thalamic lesions. The present experiment allowed these subsidiary comparisons to be made.

\section{Materials and Methods}

Subjects. Sixteen experimentally naive male cynomolgus monkeys ( $\mathrm{Ma}$ caca fascicularis) were used in the experiment. At the time of surgery (see below) their averaged weight was $4.4 \mathrm{~kg}$. Before beginning the experimental task described below, they were first trained to touch patterns displaycd on the television screen. This preliminary training, including magazine training and autoshaping, was identical to that described by Gaffan et al. (1984, p 202).

Surgical groups. Upon completing preoperative training as described below, the animals were divided into 5 surgical groups. Nine animals, constituting 3 groups of 3 animals each, were given bilaterally symmetrical lesions. One group had bilateral amygdalectomy (group A), another had bilateral lesions in the ventromedial prefrontal cortex (group $\mathrm{VF}$ ), and a third had bilateral lesions of the medial portion of the inediodorsal thalamus (group MD). The remaining 2 groups were disconnection groups in which crossed unilateral lesions were produced either in the ventromedial prefrontal cortex and the amygdala (group
$\mathrm{VF} / \mathrm{A} ; n=4$ ) or in the mediodorsal thalamus and the amygdala (group $\mathrm{MD} / \mathrm{A} ; n=3$ ). All of the animals in the disconnection groups were first given one unilateral lesion alone and were tested behaviorally after this first surgery (Post 1) in order to verify that a single unilateral lesion was without effect in the present task. They were then given a second lesion, contralateral to the first, and a further behavioral test (Post2) was administered. All 3 animals in group MD/A had the lesion in the mediodorsal thalamus first and the lesion in the amygdala second. Among group VF/A, 2 animals (D1 and D2) had the ventromedial prefrontal lesion first, while the other 2 (D3 and D4) had the amygdala lesion first. Finally, group VF/A was subjected to forebrain commissurotomy and were given a third behavioral test (Post3).

Surgical methods. The surgical operations were carried out under sterile conditions. Barbiturate was infused through an intravenous cannula at a rate sufficient to maintain deep anesthesia. The ablations were made by aspiration under visual guidance with the aid of an operating microscope. The tissue was closed in layers with silk sutures. A prophylactic dose of antibiotic was administered at the end of the surgery. Postopcrative rccovery was in all cases uneventful.

The surgical method for making amygdala lesions was identical to that described by Gaffan and Harrison (1987). The medial temporal lobe was exposed by retracting the frontal lobe over the orbit. The pia mater was cauterized in an area approximately $5 \mathrm{~mm}$ in diameter on the medial surface of the temporal lobe medial and superior to the rhinal sulcus. The amygdala and the periamygdaloid cortex medial to the amygdala were then ablated by aspiration through the defect in the pia mater. White matter of the temporal stem, and the lateral ventricle and anterior surface of the hippocampus, were visible boundaries of the ablation posteriorly, laterally, and inferiorly.

To produce an ablation in the ventromedial frontal cortex, a bone flap was raised over the frontal lobe, and the dura mater was cut in a $Y$ shape to expose the whole of the dorsolateral surface of the frontal lobc. The lobe was then retracted from the orbit in order to expose its inferior surface. The pia mater was cauterized along the medial bank of the lateral orbital sulcus, and this line of cautery was extended posteriorly to the lateral sulcus and anteriorly to the lip of the inferior convexity. The line of cautery was further continued first along the lateral sulcus in a medial direction, then along the cortex adjacent to the olfactory tract at the most medial part of the inferior convexity, and finally along the lip of the inferior convexity to meet the original cautery along the line of the lateral orbital sulcus. The cortex and pia mater bounded by this continuous line of cautery was then removed by aspiration. Finally, the lesion was extended by aspiration into the cortex in the inferior part of the medial wall of the hemisphere, the intention being to extend the lesion to the level of the rostral sulcus, which, however, was not prominent in most cases. The dura mater was sewn, and the wound was closed in layers.

For the ablations in the mediodorsal thalamus, the method employed was similar to that described by Aggleton and Mishkin (1983). The right hemisphere was exposed and retracted over the splenium of the corpus callosum. The splenium was cut over the midline with a glass aspirator. The membrane covering the thalamus was cut in the midline by applying cautery in order to expose the posterior commissure, the third ventricle, and the posterior $5 \mathrm{~mm}$ of the midline thalamus. Next, the most posterior $5 \mathrm{~mm}$ of the massa intermedia of the thalamus was cut in the midline with a glass aspirator. Tissue adjacent to the cut massa intermedia was removed by aspiration with a metal aspirator, either bilaterally (group MD) or on 1 side only (group MD/A). Approximately 2 $\mathrm{mm}$ of tissue lateral to the midline was removed. The lesion extended $5 \mathrm{~mm}$ anterior from the posterior end of the thalamus.

For forebrain commissurotomy in group VF/ $\Lambda$, the methods werc similar to those previously described (Gaffan and Harrison, 1987). The corpus callosum and the anterior commissure were sectioned in the midline.

Histology. The animals were deeply anesthetized, then perfused through the heart with saline followed by formol-saline solution. The brains were blocked in the coronal stereotaxic plane posterior to the lunate sulcus, then removed from the head, photographed, and allowed to sink in a sucrose formalin solution. The brains were cut in 50- $\mu \mathrm{m}$ sections on a freezing microtome. Every fifth section was retained and stained with Cresyl violet. The series of sections from each animal was examined microscopically.

Group $V F$. The lesions in this group were as intended. The cortex on the orbital surface extending medially from the lateral orbital sulcus was removed bilaterally. The lateral bank of the lateral orbital sulcus 


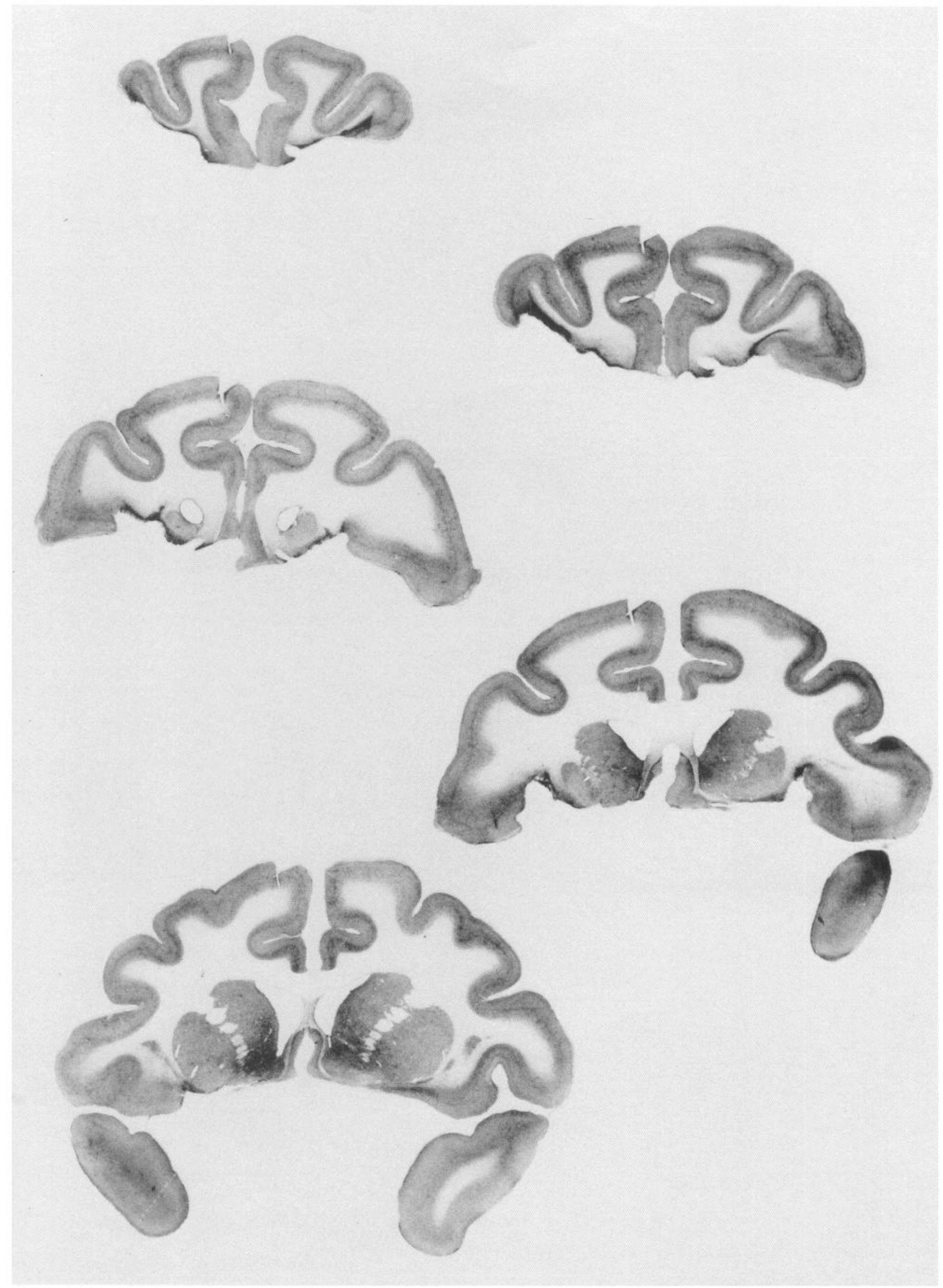

Figure 1. Five sections, $2 \mathrm{~mm}$ apart, through the lesions in animal VF3. 


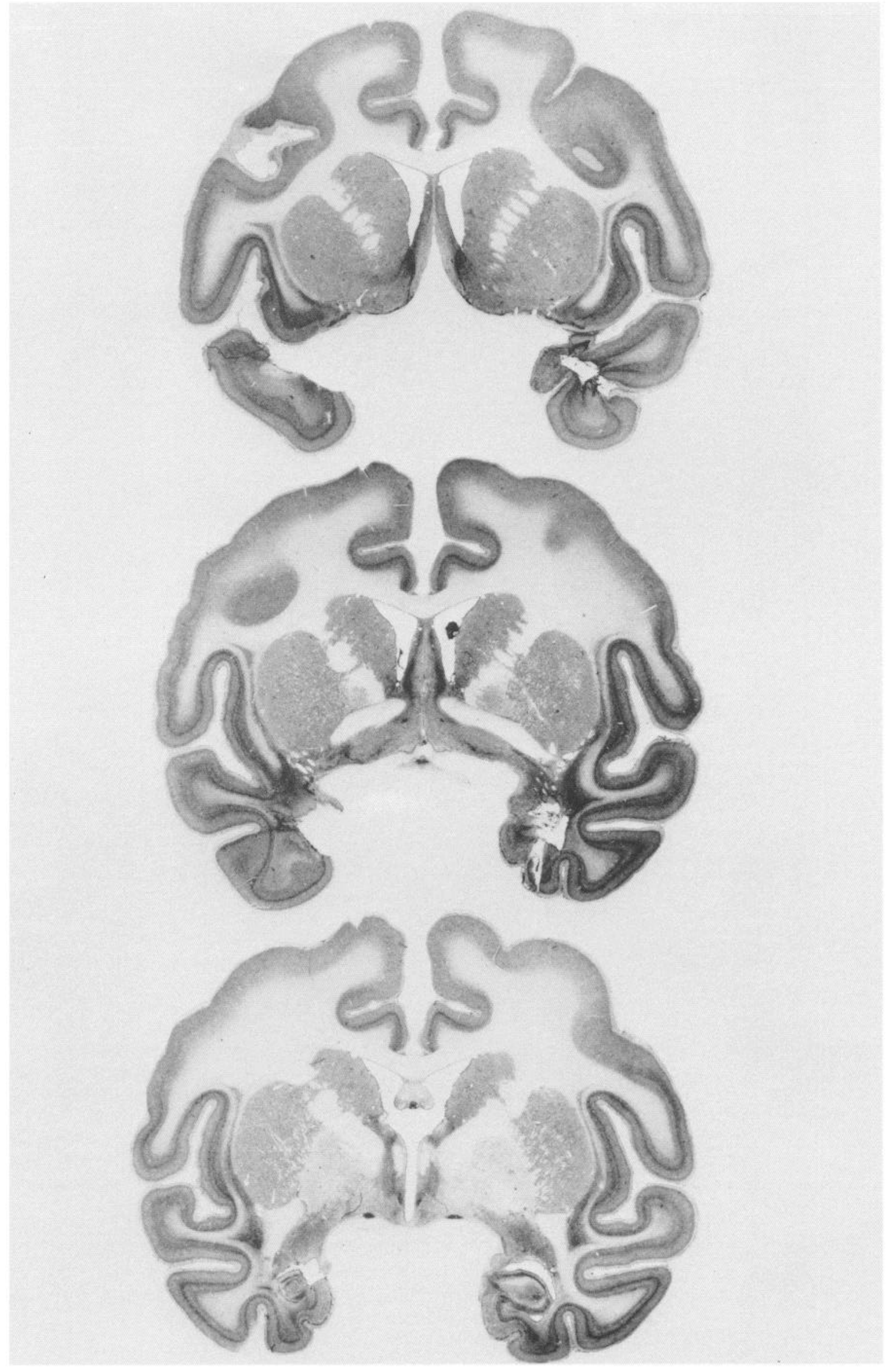

Figure 2. Three sections, $2 \mathrm{~mm}$ apart, through the lesions in animal A3. 
and the inferior convexity of the frontal lobe and its dorsolateral surface were intact. On the medial wall of the hemisphere, the most inferior 3$5 \mathrm{~mm}$ of cortex was ablated, but the rest was intact. A series of sections from a representative animal in this group is shown in Figure 1.

Group $A$. The bilateral amygdalectomies in this group were similar to the unilateral amygdalectomies in the study by E. A. Gaffan et al. (1988). The amygdala removals were complete except for small parts of the posterior dorsal amygdaloid complex in some animals. A series of sections from a representative animal in this group is shown in Figure 2.

Group $M D$. The terminology we have adopted for describing the posterior thalamus is taken from Olszewski (1952), modified in accordance with Burton and Jones (1976) and Asanuma et al. (1983). The term rhomboid nucleus (Berman and Jones, 1982) is used to refer to the collection of areas designated by Olszewski (1952) as parts of the central nucleus (e.g., superior central nucleus, densocellular central nucleus, intermediate central nucleus). The region Olszewski refers to as the inferior central nucleus is termed the central medial nucleus (see Jones, 1985).

In order to facilitate identification of nuclear boundaries in the animals with bilateral MD lesions, sections adjacent to those stained with Cresyl violet were fiber stained by a modified Gallyas method. To aid the reconstruction of the lesions in this group, we first prepared drawings of a series of 8 sections at $1-\mathrm{mm}$ intervals taken through the posterior thalamus of a cynomolgus monkey in which the posterior thalamus was intact. (This animal had received a fornix transection in the course of a different experiment.) The brain of this animal had been processed in the same manner as those of the animals with bilateral MD lesions in the present experiment. The lesions in the MD group were illustrated by plotting them onto the drawings of the intact posterior thalamus. Only 6 sections are illustrated (Fig. 3), because no significant amount of damage in the MD animals was observed in the most anterior and most posterior of the 8 sections used. Drawings of the 6 intact sections are shown in Figure 3 next to photographs of the Nissl-stained material from the animal on which they are based.

Figure 4 shows photomicrographs of Nissl-stained coronal sections through the lesion in animal MD1 at 2 of the levels (numbers 4 and 5) that are illustrated in Figure 3. The reconstruction of this animal's lesion, plotted onto our drawings of the intact thalamus, is shown in Figure 5 (top panel). Figure 5 (lower panel) and Figure 6 (top panel) show the lesions in animals MD2 and MD3, reconstructed in the same way.

All 3 animals in the MD group had extensive bilateral lesions in the magnocellular medial region of the mediodorsal nucleus (MDmc). The largest lesion was in animal MD3, with only slight sparing of the most caudal portion. The lesions in MD1 and MD2 left a small amount of the rostral MDmc intact and spared the most caudal portion. The parvocellular lateral portion of the mediodorsal nucleus was damaged in all cases, but the damage was minor except in the right hemisphere of animal MD2, where an infarction resulted in more extensive gliosis and cell loss. Damage outside the MDmc was similar in the 3 animals: Among the intralaminar thalamic nuclei, there was extensive damage to the rhomboid nucleus and the central medial nucleus, but the parafascicular and center median nuclei were damaged only in their medial parts. Other nuclei of the midline thalamus, including the parataenial and reuniens, were also damaged, and the paraventricular nucleus of the epithalamus was removed for the entire anteroposterior extent of the lesions. The anterior thalamus appeared normal in each of the 3 brains. All 3 animals had the sagittal section of the corpus callosum and hippocampal commissure overlying the posterior thalamus, and some gliosis was observed in the fimbria and fornix.

Group $M D / 4$. The unilateral amygdala lesions in this group were similar to those made bilaterally in group A. The unilateral MD lesions in this group were reconstructed in the same way as in the MD group (described above). Figures 6 and 7 show the individuals' unilateral lesions. Damage to the midline thalamic nuclei and to the corpus callosum was similar in the MD/A group to that observed in the MD group, but the MDmc was left intact unilaterally in the MD/A group.

Group $V F / A$. The unilateral amygdala and ventromedial prefrontal lesions in this group were similar to those made bilaterally in groups $\mathrm{A}$ and VF. In addition, the corpus callosum and anterior commissure were sectioned in all cases. A series of sections from a representative animal is shown in Figure 8.

Apparatus and stimuli. The computer-controlled apparatus, described in detail by Gaffan et al. (1984), displayed pairs of complex patterns against a plain gray background on a television screen. The stimuli were presented $90 \mathrm{~mm}$ to the left and right of the screen's center. The monkey reached out through the bars of a transport cage to touch stimuli on the screen. Infrared beams crossing the surface of the screen detected the animal's touches. Each of the 2 visual stimuli that constituted a discrimination problem was made of a small shape superimposed on a larger shape (approximate height, $20 \mathrm{~mm}$ and $40 \mathrm{~mm}$, respectively) Each of the shapes was taken at random from a predefined set of 127 symbols and was displayed in a color chosen at random from 255 possibilities. The random-number generator that determined stimuli for each problem and their left-right position at every trial was seeded with the session number, so the stimulus sequence was unique to each session, but was the same in a given numbered session for all monkeys.

Food rewards were dispensed into a bowl just in front of the lower edge of the screen. The rewards were either peanuts, sugar-coated puffed rice, or banana-flavored pellets, according to the preference of the monkey. The only visible illumination in the experimental cubicle came from the television screen. The background color of the screen, against which stimuli were displayed, was a medium gray that shed enough light by which to see. An infrared light source allowed the monkey to be watched over closed-circuit television.

Task. Each daily session had 5 new visual-discrimination problems presented serially. For each new problem, a positive and a negative stimulus were chosen at random from the large population of available stimuli (see Apparatus, above). The problem was presented for 20 consecutive trials. The intertrial interval was $10 \mathrm{sec}$ throughout. For each trial within a problem, the 2 stimuli were presented simultaneously, occupying the left and right positions at random, and the animal chose 1 stimulus by touching it. The stimulus not chosen then immediately disappeared from the screen, while the chosen stimulus blinked off once $(20 \mathrm{msec})$, then on again for $0.5 \mathrm{sec}$. During the $0.5-\mathrm{sec}$ redisplay of the chosen stimulus, a food reward was dispensed if the chosen stimulus was the positive one.

Training schedule. After initial shaping, as described in Subjects, above, the animals were given 45 preoperative sessions ( 225 new discrimination problems) of the task described above. Following each surgery, 20 sessions ( 100 new problems) of the same task were given.

\section{Results}

To measure the effect of surgery, speed of learning was assessed for each animal in the last 5 sessions ( 25 problems) of each postoperative behavioral test and compared with that animal's speed of learning in the last 5 sessions ( 25 problems) given preoperatively. Data from the early postoperative sessions gave qualitatively similar results to those obtained from the last 5 sessions, but with greater variance among individual animals within groups. The method we have adopted, that of giving 20 postoperative sessions and basing our conclusions primarily on the last 5 of the postoperative sessions, is the same as that adopted in several recent similar studies (e.g., Gaffan and Harrison, 1987; E. A. Gaffan et al., 1988; Gaffan et al., 1989). We have generally observed that the first few postoperative sessions can give highly variable results, and this is understandable given the variable reactions of individual animals to a prolonged break in daily behavioral testing, with or without surgery.

Figure 9 shows data from an individual animal in order to illustrate how the efficiency of learning was measured. When errors (choices of the negative stimulus) are plotted against trial position within a problem and averaged over 25 problems, it

Figure 3. Six sections, $1 \mathrm{~mm}$ apart, through the intact posterior thalamus of a monkey not taking part in the present experiment. Next to each section is the drawing of that section, on which the lesions were reconstructed in groups MD and MD/A (see Figs. 5-7). For definitions of abbreviations, see Appendix. Scale bars, $2 \mathrm{~mm}$. 

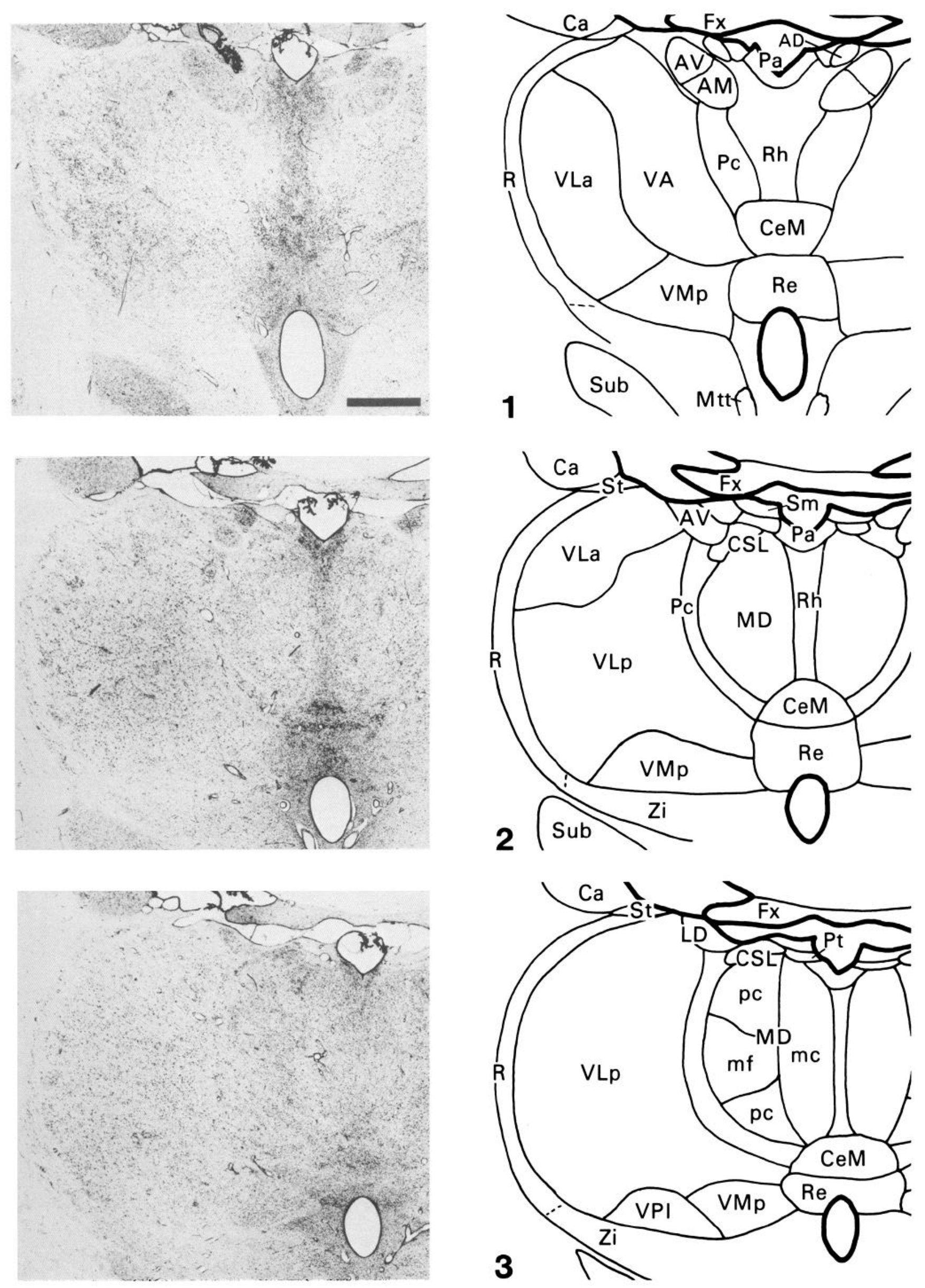

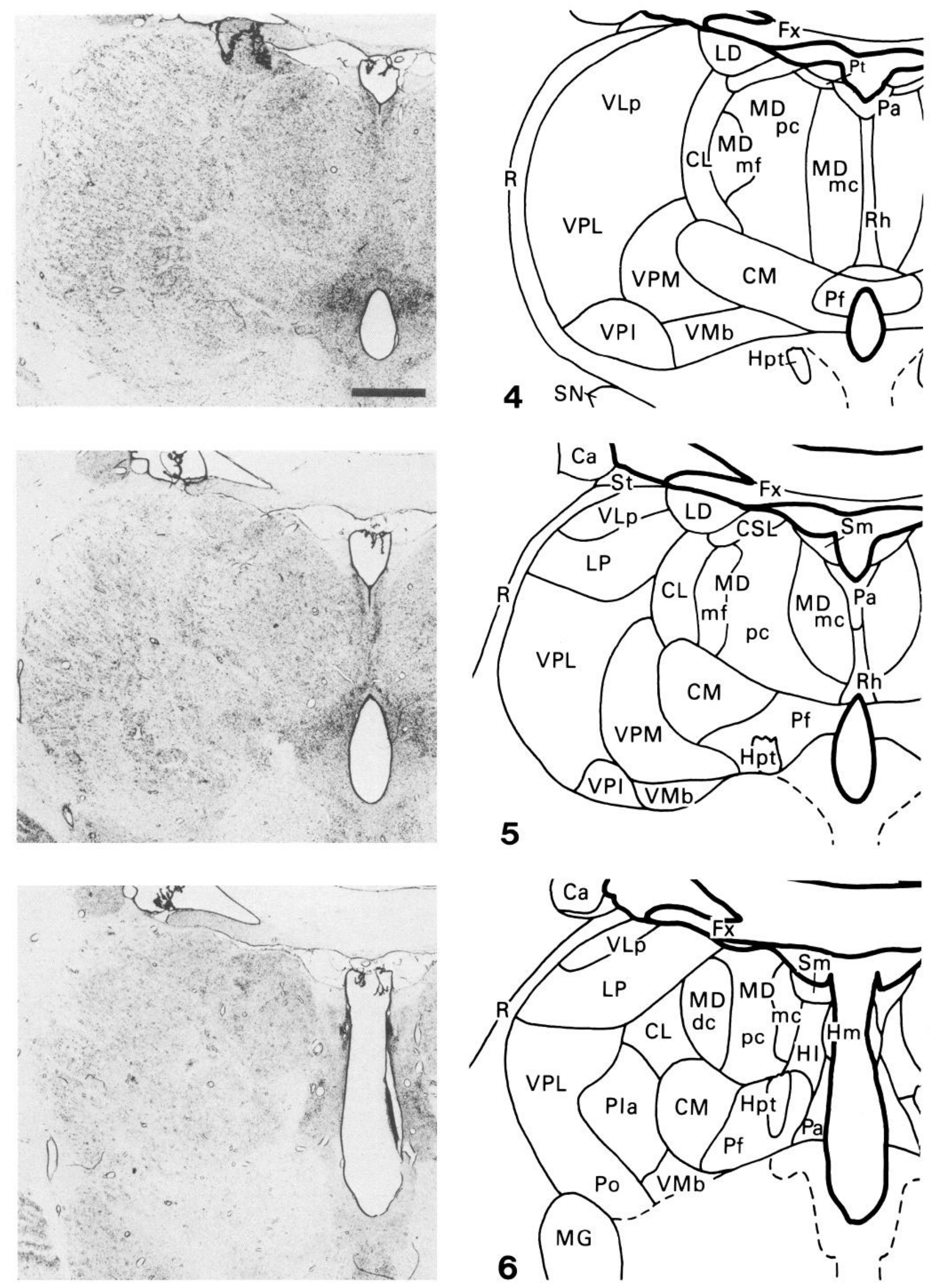


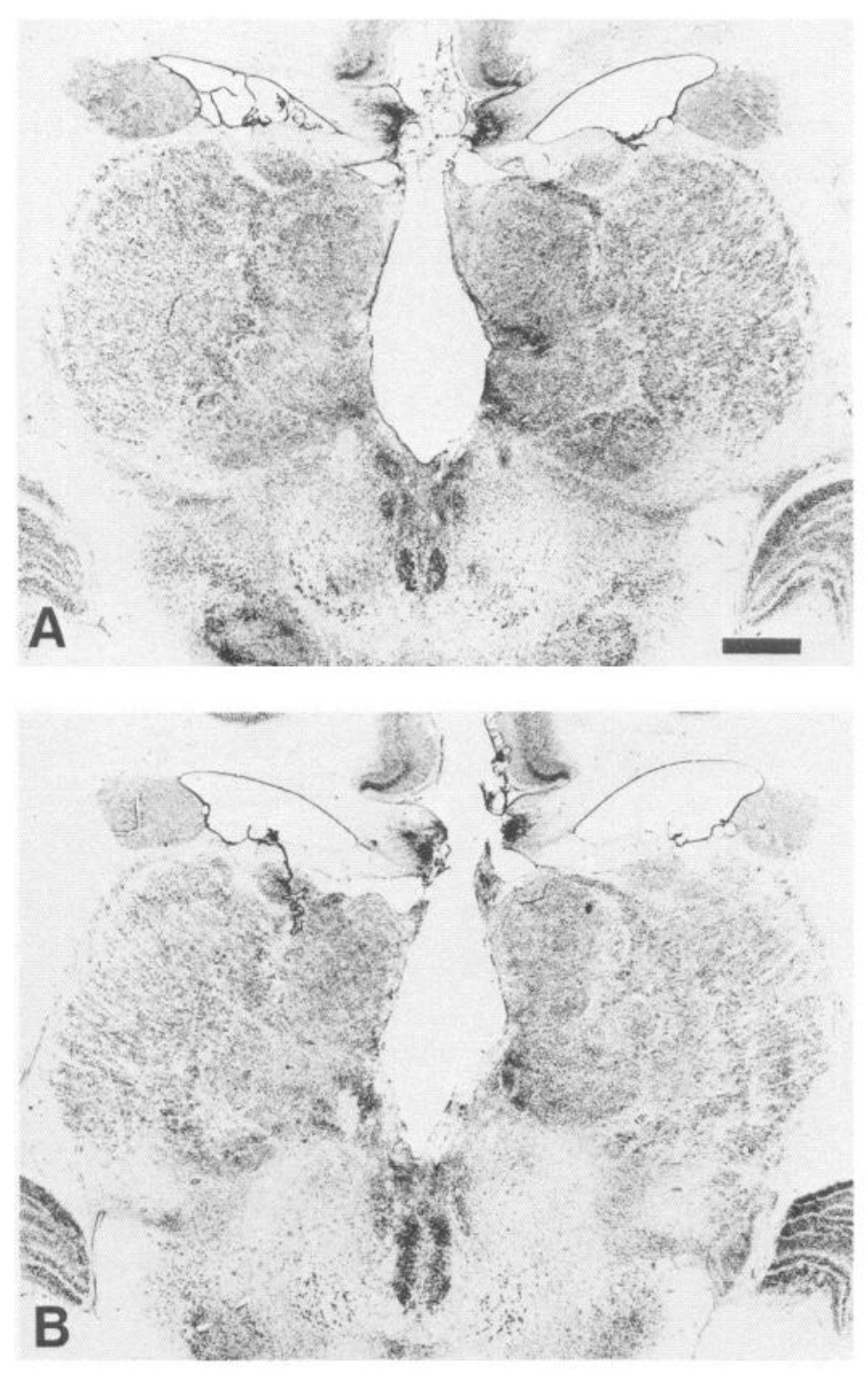

Figure 4. Two sections, $1 \mathrm{~mm}$ apart, through the lesion in animal MD1. Scale bar, $2 \mathrm{~mm}$.

\begin{tabular}{llrl}
\hline \multicolumn{2}{l}{ Table 1. } & Results from the animals with bilateral symmetrical lesions \\
Group & Animal & Pre & Post \\
\hline A & A1 & 20.0 & 35.4 \\
& A2 & 6.9 & 25.7 \\
\multirow{2}{*}{ Mean } & A3 & 7.6 & 22.3 \\
MD & & 11.5 & 27.8 \\
& MD1 & 7.6 & 16.8 \\
& MD2 & 19.8 & 37.1 \\
Mean & MD3 & 11.8 & 48.2 \\
VF & & 13.1 & 34.0 \\
& VF1 & 9.5 & 28.6 \\
& VF2 & 10.3 & 20.4 \\
Mean & VF3 & 9.1 & 27.8 \\
& & 9.6 & 25.6
\end{tabular}

Each cell under Pre and Post shows percent error in T2-20 of 25 new discrimination problems.

\begin{tabular}{|c|c|c|c|c|c|}
\hline Group & Animal & Pre & Post 1 & Post2 & Post3 \\
\hline \multirow[t]{4}{*}{ VF/A } & VF/A1 & 6.5 & 9.9 & 8.8 & 7.2 \\
\hline & VF/A2 & 5.7 & 10.5 & 15.2 & 19.6 \\
\hline & VF/A3 & 12.8 & 12.8 & 25.1 & 21.1 \\
\hline & VF/A4 & 8.2 & 6.3 & 17.9 & 20.0 \\
\hline Mean & & 8.3 & 9.9 & 16.8 & 17.0 \\
\hline \multirow[t]{3}{*}{$\mathrm{MD} / \mathrm{A}$} & $\mathrm{MD} / \mathrm{A} 1$ & 14.9 & 13.7 & 19.8 & - \\
\hline & MD/A2 & 10.9 & 13.3 & 19.4 & - \\
\hline & $\mathrm{MD} / \mathrm{A} 3$ & 9.9 & 9.7 & 13.1 & - \\
\hline Mean & & 11.9 & 12.2 & 17.4 & - \\
\hline
\end{tabular}

Each cell shows percent error in T2-20 of 25 new discrimination problems.

can be seen that the animal begins each problem at chance on average ( 50 percent errors), then reduces the rate of errors as learning proceeds. Thus the error rate in trials 2-20 (T2-20) falls quickly if learning is efficient, but slowly if learning is poor. Therefore, the average rate of errors in T2-20 can be taken as an index of the animal's speed of learning. Figure 9 shows the animal's learning curve before and after operation and also shows, on the same scale, the average percent error for T2-20 pre- and postoperatively. Finally, the figure shows the difference (D) between the pre- and postoperative T2-20 averages. This difference was the measure of each animal's deficit. The deficit is the decline in the animal's speed of learning as a result of surgery.

Tables 1 and 2 show the mean percent error for T2-20 for each animal at each surgical stage of the experiment. Figure 10 shows the deficits for the groups and the individuals. Hence, in the case of animal A3, whose data is shown in Figure 9, the averages shown in Figure 9 as the bars labeled T2-20 Pre and Post appear in Table 1 as the percentage error rates 7.6 and 22.3 , and the difference between these 2 error rates, 14.7, appears as the bar labeled D in Figure 9 and as the lowest of the 3 dots in group A in Figure 10.

All the animals with bilaterally symmetrical lesions (groups $\mathrm{A}, \mathrm{MD}$, and VF) showed substantial postoperative impairments. Among the animals in the disconnection groups (MD/A and $\mathrm{VF} / \mathrm{A}$ ), it should first be noted that a single unilateral lesion did not substantially affect learning rates (Table 2, Post1). However, the addition of a crossed asymmetrical lesion resulted in consistent deficits (Post2). Figure 10 shows the disconnection deficits (Post2 minus Pre), as well as the deficits of the groups with bilaterally symmetrical lesions.

To assess the statistical significance of the comparisons shown in Figure 10, we computed designed comparisons using the pooled within-group error term with 11 degrees of freedom. Overall, the effect of the bilaterally symmetrical surgeries was more severe than the effect of the disconnection surgeries $(F=$ 9.237; $d f=1,11 ; p<0.02$ ). There was no significant difference among the 3 different bilaterally symmetrical surgeries $(F<1$; $d f=2,11)$ and no significant difference between the 2 disconnection surgeries $(F<1 ; d f=1,11)$. The bilaterally symmetrical surgeries, on average, produced a significant positive deficit, that is, significantly greater than $0(F=59.788 ; d f=1,11 ; p<0.01)$, and the disconnection surgeries, on average, similarly produced a significant positive deficit $(F=7.656 ; d f=1,11 ; p<0.05)$. Therefore, we conclude that both bilaterally symmetrical surgeries and disconnection surgeries produced a deficit, that the 

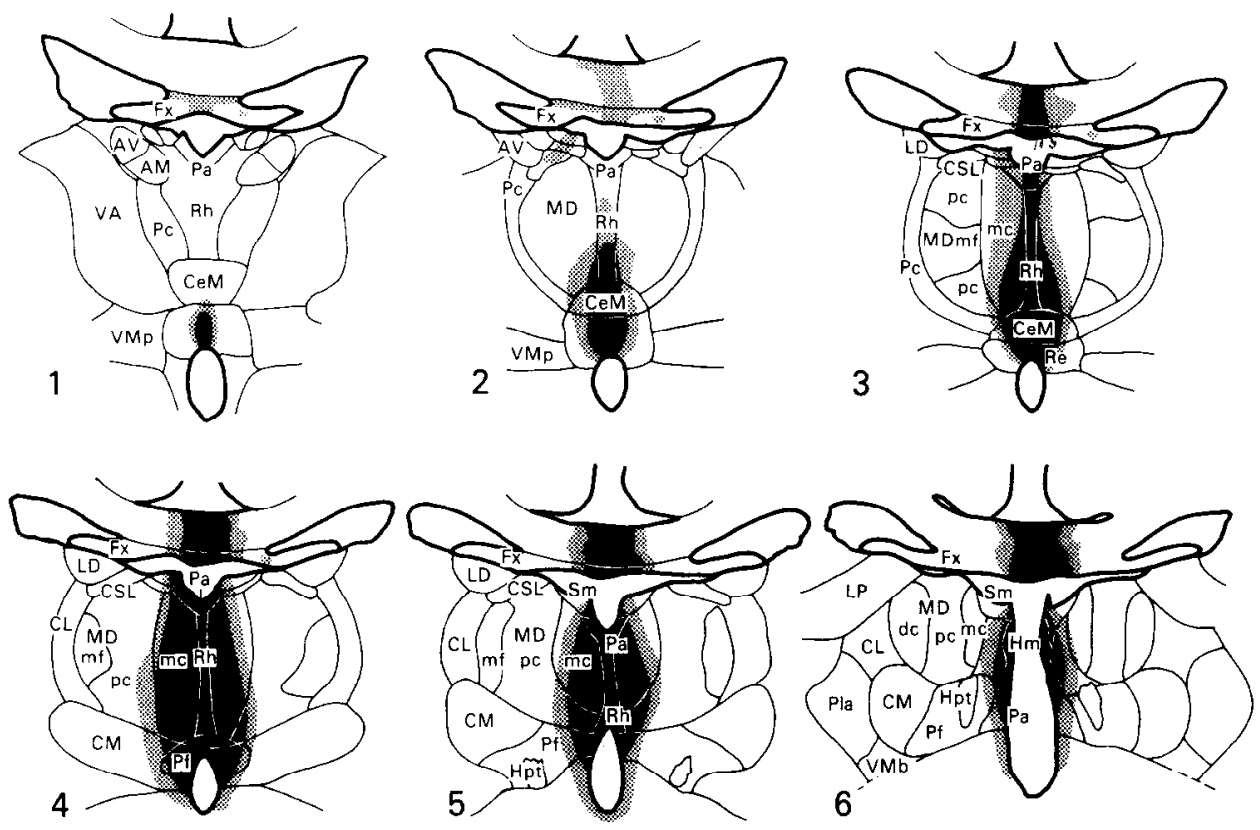

MD1
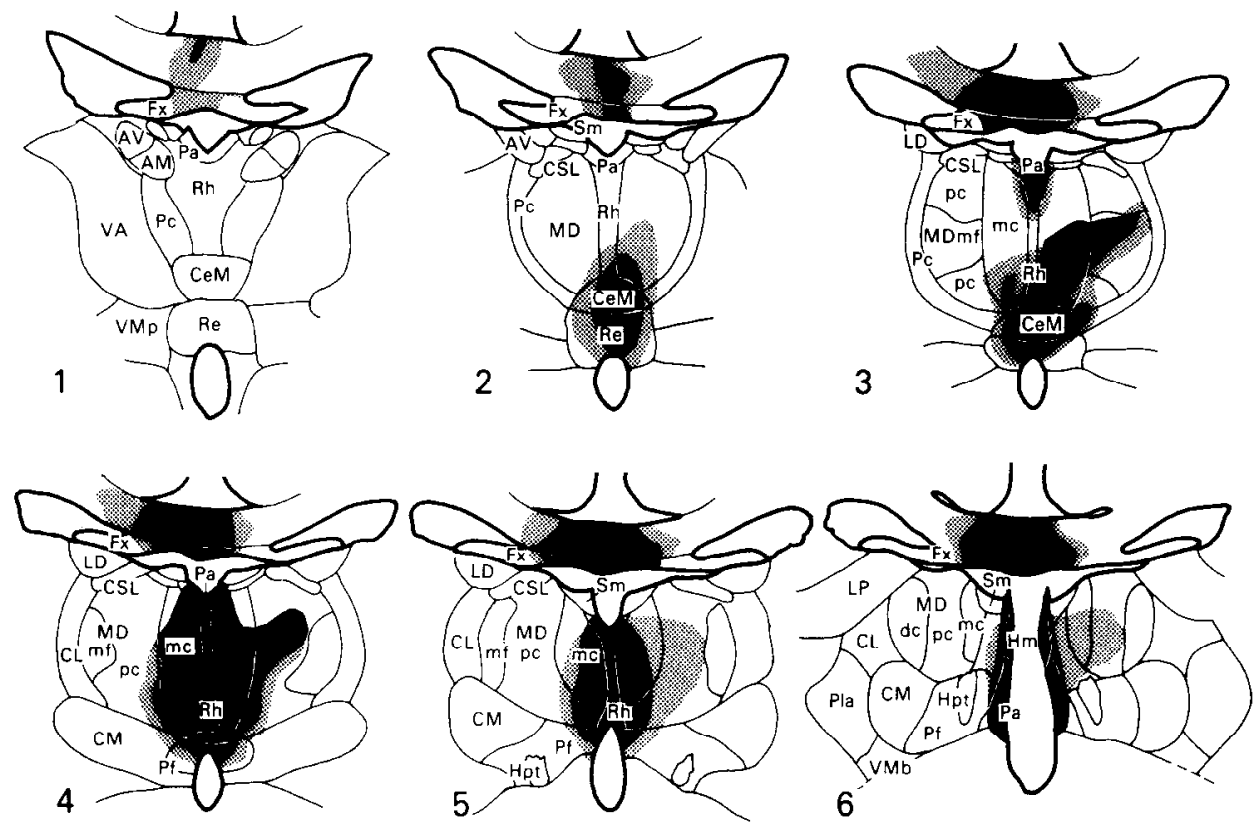

MD2

Figure 5. Reconstructions of the lesions in animals MD1 (upper panel) and MD2 (lower panel). The 6 levels shown are $1 \mathrm{~mm}$ apart. For definitions of $a b$ breviations, see Appendix. bilaterally symmetrical deficit was more severe than the disconnection deficit, and that there was no evidence of differences in severity between the different kinds of bilaterally symmetrical surgery or between the different kinds of disconnection surgery.

The Post 3 scores in Table 2 show that the addition of forebrain commissurotomy did not exacerbate the impairment of the VF/A group.

Within each surgical group, individual animals' deficit scores were compared with their histological results in order to assess the possibility that behavioral variation between individuals within groups could be explained by variation in the extent of the lesions. In the MD group, it is noticeable that the animal with the largest deficit, MD3, also had the largest lesion (see Fig. 6). In group VF, the animal with the smallest deficit, VF2, was not found to have any less damage than the other 2 animals, whose scores were similar to each other. In the $\mathrm{A}$ and $\mathrm{MD} / \mathrm{A}$ groups, there was little variation among individual animals' behavioral results. In the VF/A group, the animal with the smallest deficit had the full intended removal (Fig. 8). Therefore, there is little evidence that variations in lesions within groups 

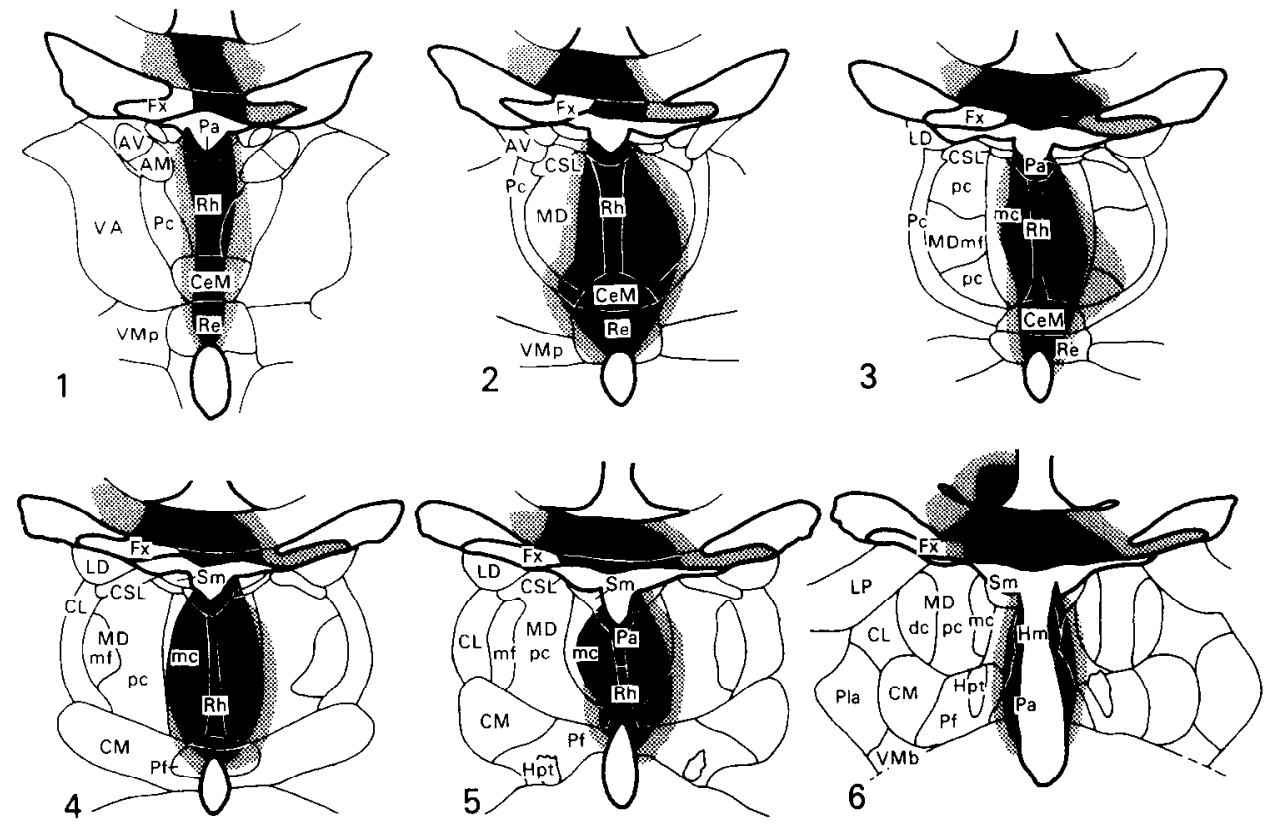

MD3

Figure 6. Reconstructions of the lesions in animals MD3 (upper panel) and MD/Al (lower panel). The 6 levels shown are $1 \mathrm{~mm}$ apart. For definitions of abbreviations, see Appendix.
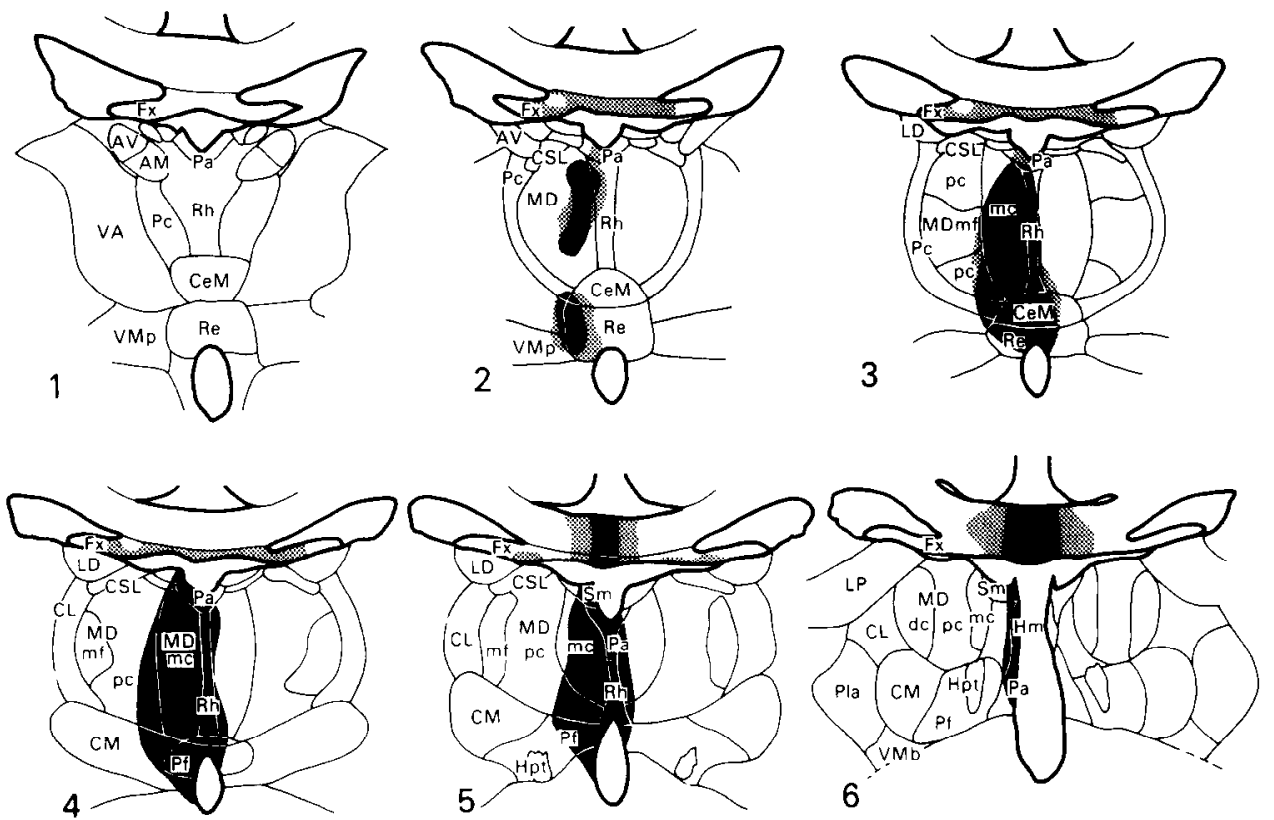

contributed to behavioral variation among individuals, apart from the suggestive case of animal MD3.

\section{Discussion}

Bilateral amygdalectomy produced a substantial and consistent deficit in the present task. This effect is in accordance with the analysis of amygdalar function in visual discrimination learning put forward by Gaffan and Harrison (1987), E. A. Gaffan et al. (1988), and Gaffan et al. (1989). In the present task, there was no local visual feedback on the monitor screen to indicate whether the animal's choice had been correct or incorrect. Instead, the most salient feedback to indicate that a choice was correct was the delivery of food to a site spatially separate from the discriminative stimulus. Thus, the conditions of the present task were similar to those in the experiment by E. A. Gaffan et al. (1988), where an impairment was observed in a task that provided no local differential visual feedback for correct choices, but were different from those in the experiment by Gaffan et al. (1989), where no impairment was observed in a task that did provide local visual feedback for correct choices. The present results from bilateral amygdalectomy thus support the idea that the importance of the amygdala for visual-discrimination learn- 

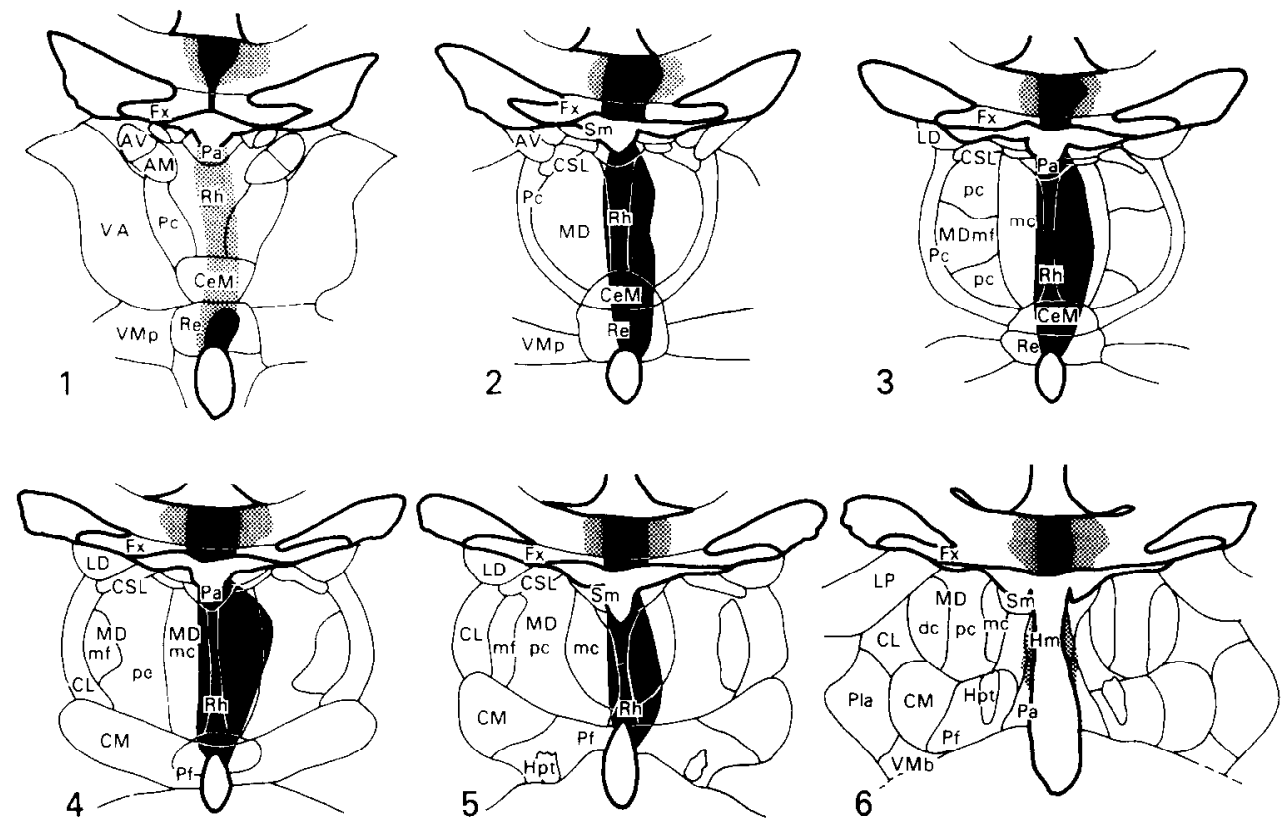

$\mathrm{MD} / \mathrm{A} 2$
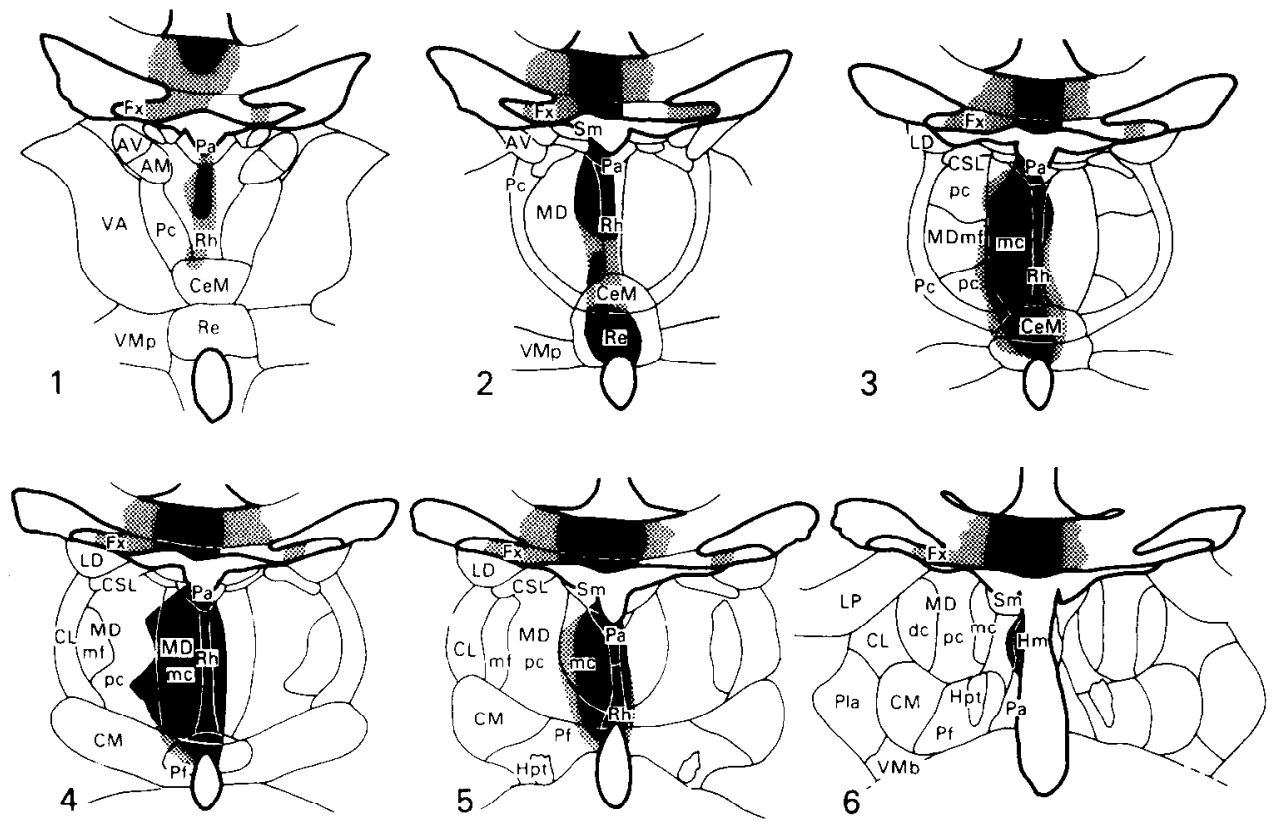

MD/A3

Figure 7. Reconstructions of the lesions in animals MD/A2 (upper panel) and MD/A3 (lower panel). The 6 levels shown are $1 \mathrm{~mm}$ apart. For definitions of abbreviations, see Appendix.

ing depends on the associative structure of the task. The amygdala is important when the discriminative stimuli are associated directly with the incentive value of the food reward, and this type of association is promoted in the normal animal when, as here, the task does not provide a spatially contiguous visual feedback associated with the visual discriminative stimuli.

For similar reasons, the effects of bilateral amygdalectomy in the present experiment are also consistent with the proposal that the amygdala is important for cross-modal associative learning. Murray and Mishkin (1985) showed that, in the task of delayed nonmatching to sample, bilateral amygdalectomy produced a much more severe deficit in cross-modal (tactile-to-visual) nonmatching than in within-modal (tactile or visual) nonmatching. They suggested that amygdalectomy might produce a general impairment in cross-modal association, an impairment that could also explain many of the affective changes that follow amygdalectomy (Murray, 1990). Association of a visual discriminative stimulus with the incentive value of a food reward is a cross-modal association, because the incentive value is presumably represented by nonvisual gustatory and affective attributes of the food. Therefore, the effects of bilateral amygdalectomy in the task studied in the present experiment are consistent with 


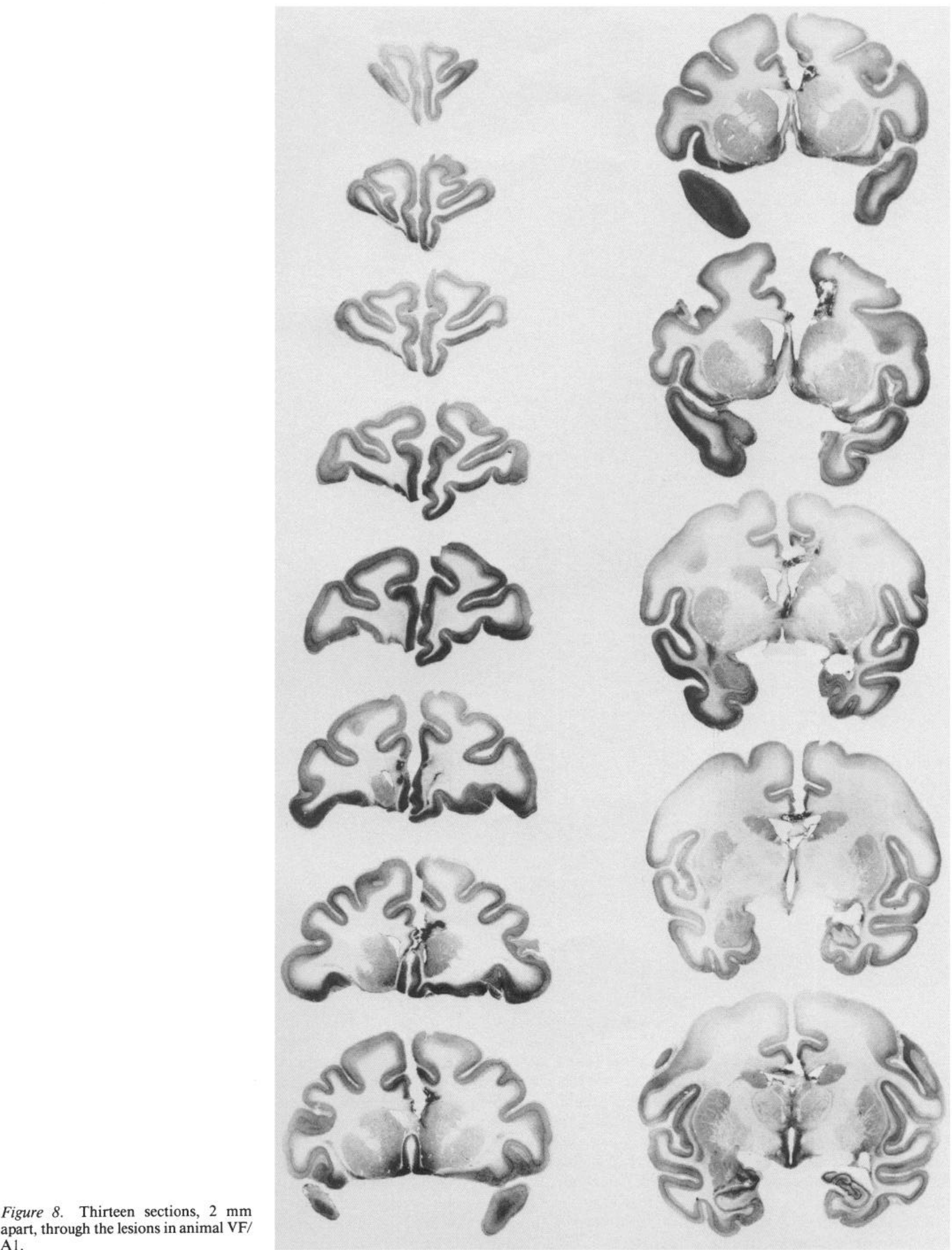




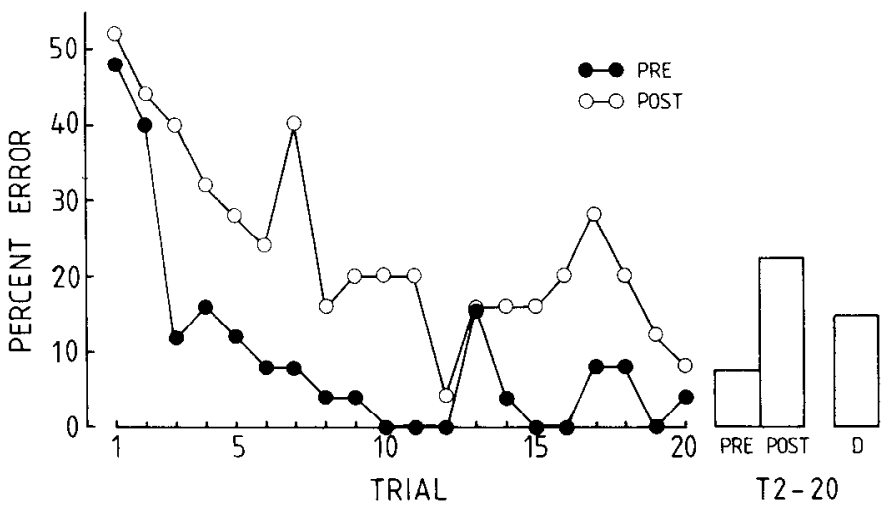

Figure 9. Results from an individual animal (A3) illustrate how the data were analyzed. The 2 learning curves on the left show withinproblem learning in the last 25 problems learned preoperatively and the last 25 learned postoperatively by this animal. On the right, the bars labeled Pre and Post show the average crror rate in T2-20 of the 2 learning curves on the left. The bar labeled $D$ shows the difference between the Pre and Post bars. For each individual in the experiment, this quantity $(D)$ was taken as a measure of the animal's postoperative deficit to be used in comparisons between the different operated groups.

Murray's (1990) analysis, as well as with Gaffan and Harrison's (1987).

Like bilateral amygdalectomy, bilateral posterior thalamic lesions (group MD) produced a severe impairment in the present task. Our results from unilateral posterior thalamic lesions, which had no consistent effect (Table 2), allow the effects of the surgery in the MD group to be attributed with confidence to their bilateral lesion in the MDmc. The unilateral lesions produced as much damage as the bilateral lesions in structures outside the mediodorsal nucleus, including the midline thalamic nuclei and the corpus callosum.

Bilateral ventromedial prefrontal lesions (group VF) also had a severe effect upon the present task, comparable to that of bilateral amygdalectomy or bilateral MD lesions. Taken together, therefore, our results from the 3 groups with bilaterally symmetrical ablations are consistent both with recent analyses of amygdalar function and with the proposition that the amygdala, mediodorsal nucleus, and ventromedial prefrontal cortex are functionally related to each other. However, the results from the disconnection groups appear to contradict the idea (outlined in the introductory remarks) that these 3 structures form a tightly linked functional pathway. Both of the disconnection groups had only mild impairments, significantly less severe than those groups with bilaterally symmetrical lesions.

It is unlikely that the mild effects in the disconnection groups can be explained by unintended sparing of tissue in their ablations. For example, animal A3 (bilateral amygdalectomy; see Fig. 2) had a less complete amygdalectomy in each hemisphere than the unilateral amygdalectomy in animal VF/Al (disconnection of amygdala from ventromedial prefrontal cortex; see Fig. 8), but animal A3 had a severe impairment similar to that of the others with bilateral amygdalectomy, while animal VF/ A1 was virtually unimpaired (see Tables 1,2 ). Similarly, the unilateral lesions of the mediodorsal nucleus showed the same range in lateral and anteroposterior extent as did the bilateral lesions (Figs. 5-7).

The groups with disconnection lesions had 2 stages of surgery, with behavioral testing after each stage. The groups with bilat-

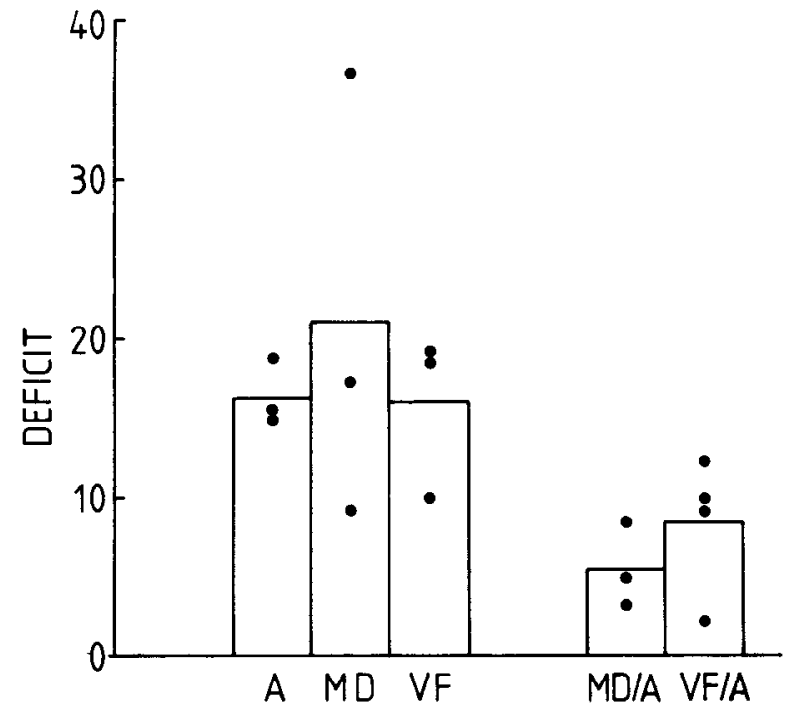

Figure 10. The postoperative deficit ( $D$ in Fig. 9) is shown for the 3 groups with bilateral lesions and the 2 groups with disconnection surgery. The bars show the group averages and the dots show the scores of individual animals.

erally symmetrical lesions, on the other hand, had a single surgical operation and postoperative test. The possibility should be considered, therefore, that this difference in the schedule of operations and behavioral tests is the cause of the relatively mild impairment in the disconnection groups as compared to the groups with bilaterally symmetrical lesions. Several results from other comparable studies suggest that 2-stage surgery is not sufficient to explain the mild effect of the disconnections in the present experiment. In an unpublished experiment still in progress, we have examined the effects of bilaterally symmetrical ablations in the amygdala, made in 2 stages of surgery with behavioral testing after each stage, on the same task as in the present experiment. Results from the first animal to complete this procedure show a deficit score (calculated as for the data presented in Fig. 10) of 30 points. This preliminary result strongly suggests that the effect of a bilaterally symmetrical lesion made in 2 stages is no less severe than that of a bilaterally symmetrical lesion made in 1 stage. Furthermore, in experiments disconnecting the amygdala from the sensory association cortex, very severe effects of disconnection have been observed following 2-stage lesions (Gaffan and Harrison 1987; E. A. Gaffan et al., 1988). E. A. Gaffan et al., studying a task similar to the present task, examined learning during trials $1-14$ of a series of visualdiscrimination problems. Choice accuracy during trials 2-14 showed an average error rate of $11.7 \%$ before disconnection surgery and an average of $33.1 \%$ after disconnection surgery (these are averages computed from the data shown in Fig. 2 of E. A. Gaffan et al., 1988). Therefore, the deficit score in that experiment, computed in a manner similar to that of the deficit scores in the present experiment (Fig. 10), was, on average, 21.4; (the difference between 33.1 and 11.7). That deficit score is similar to those obtained from bilaterally symmetrical lesions in the present study. If the amygdala was as intimately linked with the posterior thalamus and prefrontal cortex as it is with sensory-association cortex, one should expect a similar severity of impairment from the disconnection groups in the present experiment. 
It is also unlikely that the mild effects in the disconnection groups can bc explaincd by intcrhemispheric transfer of information. For example, it could be suggested that an intact amygdala in the left hemisphere could transfer information to an intact ventromedial prefrontal cortex in the right hemisphere by some route that would remain intact even after the left ventromedial prefrontal cortex and the right amygdala had been removed. One such possible route would be via interhemispheric heterotopic cortico-cortical connections, but this possibility is ruled out by our finding that the effects of disconnection were not exacerbated by forebrain commissurotomy. Another possible route would be by interhemispheric subcortical connections. Although there is some evidence that the amygdala projects into the contralateral as well as the ipsilateral thalamus (Aggleton and Mishkin, 1984), the contralateral projection is slight and may well pass through the area that was ablated in our unilateral thalamic lesions. Therefore, this route also appears an unlikely explanation of our results. The anatomical evidence reviewed in the introductory remarks, supporting the idea of a tightly linked functional pathway from the amygdala to the ventromedial prefrontal cortex via the thalamus, indicates a very heavy predominance of ipsilateral connections at every stage in the pathway. Furthermore, the powerful effects of disconnecting the visual-association cortex from the amygdala, strongly contrasting with the results from the present disconnection groups, were obtained without any commissurotomy (E. A. Gaffan et al., 1988).

For all these reasons, we conclude that the mild effects of disconnection surgery in the present groups disconfirm the simple hypothesis we outlined in the introductory remarks. This was the hypothesis that memory information retrieved by the interaction of the visual cortex with the amygdala is passed on from the amygdala to the prefrontal cortex via the thalamus in a serially organized functional pathway. One alternative possibility to be investigated in future experiments is that this type of memory information is passed on directly from the amygdala not only to the structures we have considered here, but also independently to some other area, such as the ventral striatum. Another possibility is that a severe impairment would be produced by doubly disconnecting the amygdala both from the mediodorsal thalamus and from the ventromedial prefrontal cortex by ablating the amygdala in one hemisphere and both the mediodorsal thalamus and the ventromedial prefrontal cortex in the other hemisphere. However, even if this double disconnection were to produce an impairment equal in severity to that of the animals with bilateral symmetrical ablations, that result would not support the hypothesis of a single serial pathway. Rather, it would suggest that 2 parallel pathways from the amygdala, to the mediodorsal thalamus and to the ventromedial prefrontal cortex, were partially independent of each other.

From the data presently available, it can be concluded that the functional relationships between the amygdala, thalamus, and prefrontal cortex are of a more diffuse nature than that envisaged in the hypothesis of a single serial pathway. We have seen that the effects of bilateral mediodorsal thalamic lesions or of bilateral ventromedial prefrontal lesions upon stimulusreward associative learning cannot be wholly explained by the fact that those lesions interrupt the pathway to those structures from the amygdala. These structures must therefore participate in functions that contribute to the efficiency of stimulus-reward associative learning but are independent of input from the amygdala. Equally, the effect of bilateral amygdalectomy in stimulus- reward associative learning cannot be wholly explained by the fact that that lesion intcrrupts the flow of information from the amygdala to the ventromedial prefrontal cortex. The output of information from the amygdala in this task must therefore be partially directed along a pathway independent of the ventromedial prefrontal cortex. This pattern of results shows that a mnemonic function such as stimulus-reward associative learning is not necessarily subserved by a single pathway of information flow through the serial, tightly linked stages of a single memory system, but can instead be subserved by multiple, partially independent, and partially overlapping pathways.

\section{Appendix}

\begin{tabular}{|c|c|}
\hline $\mathrm{AD}$ & Anterodorsal nucleus \\
\hline AM & Anteromedial nucleus \\
\hline AV & Anteroventral nucleus \\
\hline $\mathrm{Ca}$ & Caudate nucleus \\
\hline $\mathrm{CeM}$ & Central medial nucleus \\
\hline CL & Central lateral nucleus \\
\hline $\mathrm{CM}$ & Center median nucleus \\
\hline CSL & Central superior lateral nucleus \\
\hline $\mathrm{Fx}$ & Fornix \\
\hline $\mathrm{Hl}$ & Lateral habenular nucleus \\
\hline $\mathrm{Hm}$ & Medial habenular nucleus \\
\hline $\mathrm{Hpt}$ & Habenulopeduncular tract \\
\hline LD & Lateral dorsal nucleus \\
\hline $\mathrm{Li}$ & Limilans nucleus \\
\hline LP & Lateral posterior nucleus \\
\hline MD & Mediodorsal nucleus \\
\hline MDdc & Mediodorsal nucleus, densocellular portion \\
\hline MDmc & Mediodorsal nucleus, magnocellular portion \\
\hline MDmf & Mediodorsal nucleus, multiformis portion \\
\hline $\mathrm{MDpc}$ & Mediodorsal nucleus, parvocellular portion \\
\hline $\mathbf{M G}$ & Medial geniculate complex \\
\hline $\mathrm{Mm}$ & Mamillary complex \\
\hline Mtt & Mamillothalamic tract \\
\hline $\mathrm{Pa}$ & Paraventricular nucleus \\
\hline Pc & Paracentral nucleus \\
\hline Pf & Parafascicular nucleus \\
\hline Pla & Anterior pulvinar nucleus \\
\hline Pli & Inferior pulvinar nucleus \\
\hline Pll & Lateral pulvinar nucleus \\
\hline Po & Posterior nucleus \\
\hline$P \mathrm{t}$ & Parataenial nucleus \\
\hline $\mathbf{R}$ & Reticular nucleus \\
\hline $\operatorname{Re}$ & Reuniens nucleus \\
\hline $\mathrm{Rh}$ & Rhomboid nucleus \\
\hline SG & Suprageniculate nucleus \\
\hline $\mathrm{Sm}$ & Stria medullaris \\
\hline SN & Substantia nigra \\
\hline St & Stria terminalis \\
\hline Sub & Subthalamic nucleus \\
\hline VA & Ventral anterior nucleus \\
\hline VLa & Ventral lateral nucleus, anterior portion \\
\hline VLp & Ventral lateral nucleus, posterior portion \\
\hline VMb & Ventral medial nucleus, basal portion \\
\hline VMp & Ventral medial nucleus, principal portion \\
\hline VPI & Ventral posterior inferior nucleus \\
\hline VPL & Ventral posterior lateral nucleus \\
\hline VPM & Ventral posterior medial nucleus \\
\hline $\mathrm{Zi}$ & Zona incerta \\
\hline
\end{tabular}

\section{References}

Aggleton JP, Mishkin M (1983) Visual recognition impairment following medial thalamic lesions in monkeys. Neuropsychologia 21: 189-197.

Aggleton JP, Mishkin M (1984) Projections of the amygdala to the thalamus in the cynomolgus monkey. J Comp Neurol 222:56-68.

Amaral DG, Price JL (1984) Amygdalo-cortical projections in the monkey (Macaca fascicularis). J Comp Neurol 230:465-496.

Asanuma C, Thach WT, Jones EG (1983) Cytoarchitectonic delinea- 
tion of the ventral lateral thalamic region in the monkey. Brain Res Rev 5:219-235.

Bachevalier J, Mishkin M (1986) Visual recognition impairment follows ventromedial but not dorsolateral prefrontal lesions in monkeys. Behav Brain Res 20:249-261.

Berman AL, Jones EG (1982) The thalamus and basal telencephalon of the cat. Madison: University of Wisconsin.

Burton H, Jones EG (1976) The posterior thalamic region and its cortical projection in New World and Old World monkeys. J Comp Neurol 168:249-302.

Gaffan D, Harrison S (1987) Amygdalectomy and disconnection in visual learning for auditory secondary reinforcement by monkeys. J Neurosci 7:2285-2292.

Gaffan D, Saunders RC, Gaffan EA, Harrison S, Shields C, Owen MJ (1984) Effects of fornix transection upon associative memory in monkeys: role of the hippocampus in learned action. Q J Exp Psychol 36B:173-221.

Gaffan D, Gaffan EA, Harrison S (1989) Visual-visual associative memory and reward-association learning in monkeys: the role of the amygdala. J Neurosci 9:558-564.

Gaffan, EA, Gaffan D, Harrison S (1988) Disconnection of the amygdala from visual association cortex impairs visual reward-association learning in monkeys. J Neurosci 8:3144-3150.

Gallyas F (1979) Silver staining of myelin by means of physical development. Neurological Res 1:203-209.
Giguere M, Goldman-Rakic PS (1988) Mediodorsal nucleus: areal, laminar and tangential distribution of afferents and efferents in the frontal lobe of rhesus monkeys. J Comp Neurol 277:195-213.

Goldman-Rakic PS, Porrino LJ (1985) The primate mediodorsal (MD) nucleus and its projection to the frontal lobe. J Comp Neurol 242: 535-560.

Gower EC (1989) Efferent projections from limbic cortex of the temporal pole to the magnocellular medial dorsal nucleus in the rhesus monkey. J Comp Neurol 280:343-358.

Jones EG (1985) The thalamus. New York: Plenum.

Murray EA (1990) Representational memory in nonhuman primates. In: Neurobiology of comparative cognition (Kesner RT, Olton, DS, eds), pp 127-155. Hillsdale, NJ: Erlbaum.

Murray EA, Mishkin M (1985) Amygdalectomy impairs crossmodal association in monkeys. Science 228:604-606.

Olszewski J (1952) The thalamus of the Macaca mulatta. Basel: S Karger.

Porrino LJ, Crane AM, Goldman-Rakic PS (1981) Direct and indirect pathways from the amygdala to the frontal lobe in rhesus monkey. $J$ Comp Neurol 198:121-136.

Russchen FT, Amaral DG, Price JL (1987) The afferent input to the magnocellular division of the mediodorsal thalamic nucleus in the monkey, Macaca fascicularis. J Comp Neurol 256:175-210.

Tobias TJ (1975) Afferents to prefrontal cortex from the thalamic mediodorsal nucleus in the rhesus monkey. Brain Res 83:191-212. 